\title{
Underestimation of hepatitis E virus seroprevalence in soldiers from the Polish Special Forces
}

\author{
Maciej Bura $^{1}$, Aleksandra Bura ${ }^{2}$, Iwona Mozer-Lisewska ${ }^{1}$ \\ ${ }^{1}$ Department of Infectious Diseases, Hepatology and Acquired Immunodeficiencies, Poznan University of Medical Sciences, Poznan, Poland \\ ${ }^{2}$ Department of Infectious Diseases, Joseph Strus Multidisciplinary City Hospital, Poznan, Poland
}

We read with great interest a recent paper by Korzeniewski et al. [1] reporting the seroprevalence of hepatitis E virus (HEV) in Polish soldiers who were participants in military operations in Asia and Africa. The Authors found the presence of anti-HEV IgG antibodies (including borderline result in one soldier) in only $7.1 \%(n=18)$ out of 253 participants of the study $(6.3 \%$ when considering a confirmatory assay).

However, we think that due to the low sensitivity of the version of the Euroimmun assay used by Korzeniewski et al. [1] the seroprevalence of HEV reported in the discussed paper is severely underestimated.

We have recently compared anti-HEV IgG prevalence in blood donors (BDs) and HIV patients from Greater Poland region, west-central Poland, with both Euroimmun (original and updated cut-offs) and Wantai tests [2]. Our results demonstrate that HEV seroprevalence significantly changes (increases) after such a re-assessment. In brief, anti-HEV IgG were found in $4.6 \%$ (original cut-off of the Euroimmun assay, identical to that used in the study by Korzeniewski et al. [1]) vs. $22.9 \%$ (lowered cut-off, as suggested by Norder et al. [3]) out of 153 BDs and in 5.8\% (original cut-off) vs. 18.3\% (lowered cut-off) out of 104 HIV patients. More importantly, in both groups, HEV seroprevalence was substantially lower when assessed with the Euroimmun vs. Wantai assay.

Low sensitivity of the older version of the Euroimmun assay in comparison to some other assays (including the highly-sensitive and partially-validated Wantai assay) was also shown by others [3-5].

Additionally, among Polish BDs who were assessed in a recent nationwide investigation [6] using the Wantai assay, HEV seroprevalence was considerably higher than in Polish soldiers serving in harsh environmental conditions in highly endemic areas of the world. It was also true when taking into account age as a factor potentially influencing the presence of anti-HEV IgG antibodies. In more details, in the study by Grabarczyk et al. [6] HEV seroprevalence in BDs aged $28-57$ varied from $42.7 \%$ in those aged $28-37$ to $60.8 \%$ in persons aged 48-57. In our view, it is highly unlikely that such a large discrepancy between BDs and soldiers reflects a real situation.
Moreover, we hypothesize that anti-HEV IgG resulting from local exposure to HEV but not detected by a non-sensitive assay (as that used by Korzeniewski et al. [1]) could be the source of protective immunity for Polish soldiers when serving in countries of Africa and Asia.

It should be noted that despite the more common symptomatic course of hepatitis E caused by human-specific HEV-1 or HEV-2 prevalent in highly endemic areas (versus HEV-3 which is predominant in industrialised countries) only a few $(n=3)$ soldiers experienced icteric disease (in all of them anti-HEV IgG antibodies were undetectable). On the other hand, 15 out of 16 HEV-seropositive soldiers had no symptoms consistent with viral hepatitis.

For all these reasons, we think that it would be very interesting to know the seroprevalence of HEV in soldiers from the Polish Special Forces evaluated after implementation of the lowered cut-off value of the Euroimmun assay or, preferably, with the use of more accurate test.

\section{REFERENCES}

1. Korzeniewski K, Osińska J, Korsak J, et al. Hepatitis E virus seroprevalence in Polish soldiers serving in harsh environmental conditions. Int Marit Health. 2018; 69(2): 137-141, doi: 10.5603/ IMH.2018.0020, indexed in Pubmed: 29939391.

2. Bura M, Michalak $M$, Łagiedo-Żelazowska M, et al. HEV seroprevalence can significantly change after re-assessment. J Med Virol. 2018; 90(5): 783-785, doi: 10.1002/jmv.25039, indexed in Pubmed: 29388686.

3. Norder H, Karlsson M, Mellgren Å, et al. Diagnostic Performance of Five Assays for Anti-Hepatitis E Virus IgG and IgM in a Large Cohort Study. J Clin Microbiol. 2016; 54(3): 549-555, doi: 10.1128/ JCM.02343-15, indexed in Pubmed: 26659210.

4. Avellon A, Morago L, Garcia-Galera del Carmen M, et al. Comparative sensitivity of commercial tests for hepatitis E genotype 3 virus antibody detection. J Med Virol. 2015; 87(11): 1934-1939, doi: 10.1002/jmv.24251, indexed in Pubmed: 25959136.

5. Al-Absi ES, Al-Sadeq DW, Younis MH, et al. Performance evaluation of five commercial assays in assessing seroprevalence of HEV antibodies among blood donors. J Med Microbiol. 2018 [Epub ahead of print], doi: 10.1099/jmm.0.000807, indexed in Pubmed: 30051802.

6. Grabarczyk P, Sulkowska E, Gdowska J, et al. Molecular and serological infection marker screening in blood donors indicates high endemicity of hepatitis E virus in Poland. Transfusion. 2018; 58(5): 1245-1253, doi: 10.1111/trf.14531, indexed in Pubmed: 29492976. 